\title{
Why are Relays not Always Good for You? Performance of Different Relay Deployment Configurations in a Heterogeneous Network
}

\author{
Jagadish Ghimire $^{1}$, Catherine Rosenberg ${ }^{1}$ and Shalini Periyalwar ${ }^{2}$ \\ ${ }^{1}$ Department of Electrical and Computer Engineering, University of Waterloo, Canada \\ ${ }^{2}$ Department of Systems and Computer Engineering, Carleton University, Canada
}

\begin{abstract}
In this paper, we study three scenarios of smallcell deployment in a heterogeneous network comprising a macro base station (MBS) and a set of small cells. The first scenario corresponds to wired backhauling and the remaining two scenarios correspond to two different ways of wireless backhauling. In one of the scenarios of wireless backhauling, the backhaul links have to compete with the user links for LTE channel resources (user-band relay scenario). In the other, an additional (mmWave) band is available exclusively for operating the backhaul links (dedicated-band relay scenario). For each of the scenarios, several different configurations based on channel allocation and node capabilities are considered. We formulate an optimization framework to model, dimension and evaluate all of these configurations. For user-band relay scenario, our results show that some configurations offer either negative or negligible throughput gains over the MBS-only case. By noting that any relay deployment's performance is upper-bounded by wired backhauling with sufficiently large capacity, the results also show that some other configurations offer very good throughput gains, with values very close to the upper-bound. The results highlight the importance of the right choice of configuration to justify the deployment of user-band relay nodes. Further, our results show that, for dedicated-band relay scenario, a small fraction of a typical mmWave bandwidth suffices to yield performance very close to the upper-bound.
\end{abstract}

\section{INTRODUCTION}

Heterogeneous Networks (HetNets) represent a new paradigm in cellular networking where a number of lowpowered base stations form small-cells within a macro cell coverage. Deployment of small-cells (SCs) within a macrocell is expected to increase the cell capacity by bringing base stations (BSs) closer to the users [1]. Connecting the small cell base stations to the macro base station (MBS) via wired backhaul links is the most common scenario (wired scenario). Such SC BSs are also called pico base stations (PBSs). In this scenario, there are three types of links, the direct links (DL) between the MBS and the User Equipments (UEs), the access links (AL) between the PBSs and the UEs, and the wired backhaul links (BL) between the MBS and the PBSs. The first two types are often referred to as user links. However, deploying wired backhaul links is not always feasible. In many circumstances, the flexibility offered by wireless backhaul links makes deploying relay nodes (RNs) an attractive alternative to the wired small cells.

3GPP LTE-A standards include HetNets with relay nodes (RNs) as an important enhancement for improving macro-cell capacity and coverage, forming the so-called relay-enhanced cellular (REC) networks. Type 1 static RN of LTE-A, as defined in [2], enhances the communication between an MBS and a UE by decoding and forwarding the data packets. These RNs form cells of their own and can be viewed as small-cell BSs with wireless backhaul links operating in the same set of bands as the user links. In other words, no additional band is available for operating the backhaul links. In this paper, we refer to such a scenario as the user-band relay scenario. Unlike in the wired scenario, the backhaul links compete with user links for the radio resources (frequency, time, and transmit power). We can construct a number of different configurations of user-band relay scenario which fundamentally differ depending on the node capabilities (number of air interfaces, directivity of each air interface, etc.) and the way in which the LTE channel resources are allocated to different links. Surprisingly only a few RN configurations are considered in LTE-A and they differ mainly in terms of whether an RN can transmit to a UE while it is receiving on its backhaul link or not (e.g., in-band and out-band [2]). It is not clear how different configurations perform with respect to each other and the wired scenario. Some recent papers have studied some LTE relay configurations [3], [4] and [5], but not in a systematic way. They show that different configurations can perform very differently. Proposing a general framework to model all these configurations of user-band relay scenario and obtain their best performances when available network processes including BS-coordination/scheduling and user association are optimized, is one of the main contributions of this paper.

A third backhauling scenario also exists where a separate band is available for operating the backhaul links. The additional band exclusively available for backhaul links can be another LTE-band or even an entirely different band. We call such a scenario as a dedicated-band relay scenario. In recent years, using the mmWave bands for wireless data links is attracting a lot of attention. However, using this resource for user links is not as straightforward, mainly due to the fact that designing hand-held UEs for this spectrum is challenging. On the other hand, utilizing this spectrum for the static backhaul links can be seen as a potential solution for tackling the spectrum scarcity problem in the wireless industry, provided that a sufficient bandwidth is available and that the propagation characteristics (path-loss, shadowing 
etc.), available transmit power and the underlying physical layer techniques support the required capacity of the backhaul links. Note that such a backhauling (unlike the user-band relay scenario) can potentially be configured to approach the performance of a wired scenario. Some existing studies including [6], focus on the physical layer aspects of mmWave wireless link. To the best of our knowledge, the literature lacks studies characterizing the feasibility of mmWave backhaul in the LTE HetNet context and their comparisons with other backhauling scenarios. Characterizing their performance under realistic channel models and comparing them with wired scenario to understand the mmWave bandwidth-demand that a typical small-cell backhauling imposes is another contribution of this paper.

These two scenarios of wireless backhauling can be viewed as two special cases of a HetNet with a given number of available bands and a given number of air interfaces (AIs) at each node. In addition to the number of bands and the number of AIs at each node, the performance of a given HetNet scenario is affected significantly by a number of important network processes. In particular, the following processes are important:

1) Channel allocation among the different types of wireless links affects the interference structure as well as communication rates of links. A number of studies highlight the importance of selecting a good channel allocation scheme in HetNets (e.g., [7]).

2) User association affects the performance of a network in an overlapped coverage scenarios like HetNets. There exists a large body of work in the literature that highlight the importance of good user association (e.g., [8], [7]).

3) Transmission coordination among BSs and user scheduling involves deciding when a BS transmits and when it does not, as well as how it schedules transmission to a user in its cell [9].

In order to obtain the best performance for a given configuration of a given scenario, we need to optimize the available network processes jointly. In [10], we formulated such a joint optimization framework for wired scenario of small-cells, which we extended in [9] to include a particular configuration of the user-band relay scenario. However, the model and the extension were limited and could not incorporate all the scenarios and their configurations that are possible with multiple bands and air-interfaces.

In this study, we formulate a joint optimization framework where we allow a wireless node to have multiple air-interfaces, allowing us to consider different relay scenarios and their configurations. The framework is based on a static system "snapshot" corresponding to a given number of users and given values of channel gains for each potential link. We only focus on the downlink with one flow per user. Under an assumption that the flows are greedy and that a user can associate to more than one BS, the framework allows us to obtain proportional fair throughput allocation by utilizing the available degrees of freedom in terms of transmission coordination, scheduling, and user association optimally. Note that, relaxing the constraint that a UE has to associate to only one BS allows us to obtain a tractable and close to optimal model [9]. Using

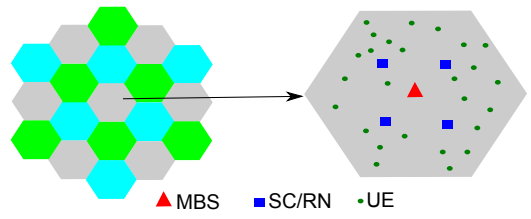

Fig. 1: Multi-cell system and a HetNet

our framework, we obtain a number of interesting engineering insights on wireless backhauling:

- Some configurations of user-band relay scenario yield very little or even negative gains whereas some others can yield performances very close to the upper bounds corresponding to the wired scenario with infinite backhaul capacities. This highlights the importance of deploying the right configurations.

- Using a dedicated band for backhauling is a promising solution for small-cells, in particular in the case of the mmWave band, since a small bandwidth is sufficient to satisfy the demand of a typical small-cell backhaul link.

\section{SYSTEM OVERVIEW}

We consider a macro-cellular layout as shown in Fig. 1, with a given inter-site distance $(I S D)$. Each macro cell (a regular hexagonal cell of length $\frac{I S D}{\sqrt{3}} \mathrm{~m}$.), in addition to a centrally placed MBS, has $X$ low-powered BSs making $X$ small cells (SCs) each at a distance $d$, placed symmetrically around the MBS (see Fig. 1). We refer to such a low-powered BS simply as a small-cell (SC). When the backhaul link of an SC is a wireless link, we call it a relay node (RN). Note that SCs are not always contained within a macro-cell (for example, [11] considers SCs located over multiple macro-cells).

We consider each macro cellular area, with its MBS, $X$ SCs, and $N$ UEs as a standalone HetNet system, and we optimize a number of network processes (channel allocation, userassociation, scheduling/coordination) within the scope of such a single macro cellular area only. However, we account for interference coming from nearby macro-cells, assuming that all interfering BSs in the nearby macro-cells are transmitting all the time. Restricting our formulation to one macro-cell level is justifiable since inter-cell resource allocations to different macro-cells are usually carried out via planning.

We assume that the user links operate on the LTE band only. The backhaul links on the other hand can either be wired, or wireless. A wireless backhaul link can operate either on the same band as the user links or on a different LTE band, or even on a non-LTE band with a different radio technology. The complexity of a two-hop wireless network with multiple bands, channels, and potentially multiple types of air-interfaces at the HetNet nodes motivates us to formulate an optimization problem that can model these complexities and details into one unified framework for throughput optimization.

\section{A GENERAL OPTIMIZATION MODEL}

Let 0 represent the MBS, $\mathcal{P}=\{1,2, \cdots, X\}$ be the set of $\mathrm{SCs}$, and $\mathcal{U}$ be the set of UEs in the macro cell under study. We focus on the downlink with a set of flows $\mathcal{F}$, where each flow $f$ originates at the MBS (node 0 ) and terminates at one of the UEs $u$. The source and destination of a flow $f$ are represented 
by $f_{s}$ and $f_{d}$ respectively. We assume that the flows are greedy. We assume that the MBS has a fixed transmit power budget of $P_{M}$ and each SC has a fixed transmit power budget of $P_{S}$. $\mathcal{N}=\{0\} \cup \mathcal{P} \cup \mathcal{U}$ represents the set of all nodes.

Let $\mathcal{B}=\{1,2, \cdots, S\}$ be the set of available bands ${ }^{1}$. Band $i \in \mathcal{B}$ is associated with its own technology (e.g., LTE) and has a number of channels $\mathcal{M}^{(i)}$, and a per-channel bandwidth $b^{i}$. We assume that at least one of them is LTE (say, Band 1) with $\mathcal{M}^{(1)}$ OFDM channels.

Each node is equipped with one or more air interfaces (AI). An air interface $m$ is associated with one of the available bands given as $B(m) \in \mathcal{B}$. A node needs to have at least one AI for each band at which it is operating. A node can have more than one AIs operating on a given band. Having multiple AIs for the same band allows a node to have multiple simultaneous links on that band. More precisely, a node with $x(x>1)$ AIs on the same band could transmit simultaneously on up to $x$ AIs in a given channel in that band. In a given channel $c$ in $\mathcal{M}^{(B(m))}$, at any given time, an $\mathrm{AI} m$ can either transmit or receive, but not simultaneously. We also assume that an AI can transmit in a set of channels of the associated band while receiving in an orthogonal set of channels of the same band. We assume that a node cannot transmit on channel $c$ in one of its AIs while receiving on the same channel in another AI. We also assume that a UE has only one AI in the LTE band. Each AI has an associated directivity. Let $D_{m}(\theta)$ be the directivity of AI $m$ on direction $\theta$. Directional AIs with very narrow beams can be used to avoid interference between AIs operating on the same set of channels.

For the ease of exposition, we logically separate an AI into a transmit $\mathrm{AI}$ (tAI) and a receive $\mathrm{AI}(\mathrm{rAI})$. A node $n \in \mathcal{N}$ contains a set of transmit AIs (tAIs) $T_{n}$ and a set of receive AIs (rAIs) $R_{n}{ }^{2}$. Let $G_{m, n}^{c}$ be the channel gain between AI $m$ and $n$ in channel $c$. Let $T$ and $R$ be the set of all tAIs and rAIs in the HetNet, respectively.

Each tAI $m \in T_{n}$ is allocated a transmit power $P_{m}$ such that $\sum_{m \in T_{n}} P_{m} \leq \bar{P}_{n}$ for all $n \in \mathcal{N}$, where $\bar{P}_{n}$ is the total power budget of node $n$ (e.g., $\bar{P}_{0}=P_{M}$ ). We focus only on the transmit power. Hence, no such power constraints exist for the rAIs.

Let $\mathcal{K}^{m} \subseteq \mathcal{M}^{(B(m))}$ be the set of channels allocated to AI $m^{3}$. We assume that the channel allocation $\mathcal{K}^{m}$ for all $m \in T \cup R$ is given. A tAI $m$ has to divide the transmit power $P_{m}$ to its channels, allocating $P_{m}^{c}$ to channel $c$, i.e.,

$$
\sum_{c \in \mathcal{K}^{m}} P_{m}^{c} \leq P_{m}, \quad \forall m \in T_{n}, \forall n \in \mathcal{N} .
$$

\section{A. Rate functions, links, and independent sets}

Band $i$ is characterized by rate functions that map channel SINR to communication rate. Let $f_{(m, n)}^{(i)}($.$) represent the$ mapping from SINR $\gamma$ between tAI $m$ and rAI $n$ in any channel $c \in \mathcal{M}^{(i)}$ to one of the supported rates $R=$ $f_{(m, n)}^{(i)}(\gamma) \in \mathcal{R}_{(m, n)}^{(i)}$ where $\mathcal{R}_{(m, n)}^{(i)}$ is the set of supported rates between tAI $m$ and rAI $n$ in band $i$. The mapping

\footnotetext{
${ }^{1}$ Even though the model allows for more bands, we study $S=1$ and 2 .

${ }^{2}$ This distinction is merely logical and hence we have $\left|T_{n}\right|=\left|R_{n}\right|$.

${ }^{3}$ In practice, there could be limits, e.g., either all or none of the channels of a given component carrier can be allocated.
}

function is determined by the available Modulation and Coding (MCS) schemes in the given band, between two AIs. Note that a band can have different rate functions for different pair of AIs (e.g., LTE backhaul links support up to 256 QAM whereas LTE user links support up to 64 QAM). $\mathcal{R}_{(m, n)}^{(i)}$ can be discrete and finite (in which case the mapping is called a discrete rate function) or it can be continuous (in which case the mapping is called a continuous rate function). For a given rate $R$, we can define the minimum required SINR as follows: $\beta_{(m, n)}^{(i)}(R)=\min \gamma$ s.t. $f_{(m, n)}^{(i)}(\gamma) \geq R$. Next, we define two notions of link: a physical link and a logical link.

A physical link is defined as a tuple $(m, n)$ where $m \in T$ and $n \in R$. Each HetNet is characterized by a set of adjacency indicators $A$. $A[j, i]$, if equal to 1 means tAI $j$ can form a physical link with rAI $i$, if equal to 0 means otherwise. For example, a tAI of one RN cannot form a link with an rAI of another RN. Also, two AIs in different bands cannot form a physical link. The set of possible/potential physical links can be defined as follows:

$$
\mathcal{L}^{P h y}=\{(m, n): m \in T, n \in R \text { s.t. } A[m, n]=1\} .
$$

For a given channel allocation $\left(\mathcal{K}^{m}\right)$ for all $m \in T \cup R$, we can define $C(\tilde{l})$ as the set of channels at which physical link $\tilde{l}=(m, n)$ operates, i.e., $C(\tilde{l})=\mathcal{K}^{m} \cap \mathcal{K}^{n}$.

Even though in the most general form, channel allocation as well as power allocation to AIs can be performed arbitrarily, we make the following assumptions to simplify the resulting optimization model.

[A1.] A physical link operates on all channels allocated to its tAI, and there is no partially overlapped channel allocation across links. i.e., if $C\left(\tilde{l}_{1}\right) \cap C\left(\tilde{l}_{2}\right) \neq \emptyset$ for some $\tilde{l}_{1}, \tilde{l}_{2} \in \mathcal{L}^{P h y}$, then we have $C\left(\tilde{l}_{1}\right)=C\left(\tilde{l}_{2}\right)$.

[A2.] Transmit power allocated to a given physical link $\tilde{l}=$ $(m, n)$, represented as $P(\tilde{l})$, equal to $P_{o(\tilde{l})}$, is equally divided among the allocated channels. So, if $p(\tilde{l})$ is the power perchannel in $\tilde{l}$, then we have $P_{m}^{c}=p(\tilde{l})=P(\tilde{l}) /|C(\tilde{l})|$ for all $c \in C(\tilde{l})$.

[A3.] Channel gains for different channels in a given physical link are equal, i.e., $G_{m, n}^{c}=G_{m, n}^{c^{\prime}}=G_{m, n}$.

Since channels have identical channel gains, and an rAI of a physical link observes the same set of interferers with identical power for all allocated channels, these assumptions make all channels of a physical link identical in terms of SINR and supported rate.

We define a logical link $l$ as a tuple $(o(l), d(l), R(l))$ where $o(l)$ is the tAI, $d(l)$ is the rAI, and $R(l)$ is its communication rate per channel. Each logical link is thus associated to a unique physical link. Let $\tilde{l}=(o(l), d(l))$ represent the physical link associated to logical link $l$. Given the set of all physical links, the set of all logical links, can be defined as follows.

$$
\begin{gathered}
\mathcal{L}^{A l l}=\left\{(o(l), d(l), R(l)): \tilde{l}=(o(l), d(l)) \in \mathcal{L}^{P h y},\right. \\
\left.R(l) \in \mathcal{R}_{\tilde{l}}^{(B(o(l)))}\right\} .
\end{gathered}
$$

In the most general form, the scheduling process in a multihop network with a given set of logical links $\mathcal{L}^{\text {All }}$ can be represented as the time-fraction $\alpha_{s}$ for which a given sub-set of logical links $s \subseteq \mathcal{L}^{A l l}$ is activated. We will call such a 
subset an independent set. Clearly, not every subset of logical links can be activated simultaneously. There are at least three fundamental limits:

1) Two links can be activated simultaneously on the same set of channels only if they do not share a tAI or an rAI.

2) SINR feasibility constraints: When a number of logical links are activated simultaneously, the SINR at each rAI should be large enough so that the signals can be decoded successfully. 3) Half-duplex communication capability: Depending on whether a tAI and an rAI of a given node are allocated the same set of channels, there is limit on whether a tAI can transmit while an rAI in the same node is receiving. For our cellular HetNet in downlink, RNs are the only nodes that could use both a tAI and an rAI. Thus, this limit is associated with the RNs only.

We are now ready to formally define an independent set (ISet) as follows.

Definition 1: For a given channel allocation $(C(\tilde{l}))$ and a given power allocation per channel $(p(\tilde{l})), s \subseteq \mathcal{L}^{A l l}$ is an ISet if the following conditions are satisfied.

$$
\begin{aligned}
& \forall l=\left(m, n, R_{l}\right) \in s: \\
& \frac{p(\tilde{l}) \cdot G_{m, n} \cdot D_{m}\left(\theta_{m, n}\right) \cdot D_{n}\left(\theta_{n, m}\right)}{N_{B(m)}+\tilde{I}_{n}+I_{l}(s)} \geq \beta_{\tilde{l}}^{(B(m))}\left(R_{l}\right) . \\
& \forall l, l^{\prime} \in s \text { s.t. } l \neq l^{\prime}: \quad o(l) \neq o\left(l^{\prime}\right) \text { and } d(l) \neq d\left(l^{\prime}\right) . \\
& \forall l, l^{\prime} \in s \text { s.t. } l \neq l^{\prime} \text { and } C(\tilde{l})=C\left(\tilde{l}^{\prime}\right): \\
& \quad \sum_{n \in \mathcal{N}} \mathbf{1}_{\left\{o(l) \in T_{n}\right\}} \mathbf{1}_{\left\{d\left(l^{\prime}\right) \in R_{n}\right\}}=0 .
\end{aligned}
$$

where $I_{l}(s)$ is given as

$$
\sum_{\substack{l^{\prime} \in s: \\ l^{\prime} \neq l, C(\tilde{l})=C\left(\tilde{l}^{\prime}\right)}} p\left(\tilde{l}^{\prime}\right) \cdot G_{o\left(l^{\prime}\right), n} \cdot D_{o\left(l^{\prime}\right)}\left(\theta_{o\left(l^{\prime}\right), n}\right) \cdot D_{n}\left(\theta_{n, o\left(l^{\prime}\right)}\right) .
$$

$\theta_{m, n}$ is the angle of AI $n$ from AI $m . N_{i}$ is the noise power per channel in band $i . \tilde{\mathcal{I}}_{n}$ is the interference from nearby macro-cells to rAI $n$ (determined by the reuse pattern). The interference is calculated by assuming that BSs in the nearby cells transmit on the allocated channels all the time.

(3) guarantees that the SINR feasibility constraints are satisfied for each logical link. (5) guarantees that the halfduplex communication constraints of the nodes are satisfied, so that a node cannot activate a tAI if one of its $\mathrm{rAI}$ is receiving in the same set of channels.

Let $\mathcal{I}^{\text {All }}$ be the set of all ISets $s \subseteq \mathcal{L}^{\text {All }}$. If $\mathcal{R}_{(m, n)}^{(i)}$ is continuous (i.e., there exists a continuous rate function) for some $m$ and $n$ in band $i=B(m)$, then $\mathcal{L}^{A l l}$ contains infinitely many links and hence it is not possible to compute $\mathcal{I}^{\text {All }}$. In order to overcome this difficulty, we define the notion of dominant ISet as follows.

Definition 2: Let $L_{P h y}(s)=\{(o(l), d(l)): l \in s\}$ be the set of physical links in ISet $s$. Then, $s \in \mathcal{I}^{\text {All }}$ dominates $s^{\prime} \in \mathcal{I}^{A l l}$ (written as $s \geq s^{\prime}$ ) if $L_{P h y}(s)=L_{P h y}\left(s^{\prime}\right)$ and $R(l) \geq R\left(l^{\prime}\right)$ whenever $\tilde{l}=\tilde{l}^{\prime}$ for all $l \in s$ and $l^{\prime} \in s^{\prime}$.

It can be shown that, for a given channel and power allocation, we can find one ISet $S_{\max }[v]$ such that $L_{P h y}\left(S_{\max }[v]\right)=$ $v$ and that dominates all ISets $s^{\prime}$ with the same set of physical links $v$.

$$
S_{\text {max }}[v]=s \in \mathcal{I}^{A l l} \text { s.t. } s \geq s^{\prime}, \forall s^{\prime} \in \mathcal{I}^{A l l}, L_{P h y}\left(s^{\prime}\right)=v .
$$

Then, from the point of view of throughput optimization, we can easily show that it is sufficient to consider only the set of dominant ISets $\mathcal{I} \subset \mathcal{I}^{\text {All }}$, which is defined as follows.

$$
\mathcal{I}=\left\{S_{\text {max }}[v]: v \subseteq \mathcal{L}^{P h y}\right\} .
$$

Note that $\mathcal{I}$ (unlike $\mathcal{I}^{A l l}$ ) is finite even if $\mathcal{R}_{(m, n)}^{(i)}$ is a continuous set. Then the set of relevant logical links can also be reduced to a finite set: $\mathcal{L}=\{l \in s: s \in \mathcal{I}\}$.

1) ON-OFF Transmission Coordination: If all of the ISets $s \in \mathcal{I}$ defined as above were allowed to be scheduled, it means that we are implicitly assuming that the MBSs and RNs perform a transmission coordination where a BS can improve the transmission rate of a physical link in another BS by occasionally pausing its own transmission. We call this an $O N-O F F$ transmission coordination among the BSs. In the LTE-A context, this is a generalization of LTE-A proposal of almost blank sub-frame (ABSF) during which the MBS does not schedule on any data channels. Let $\mathcal{I}^{O}=\mathcal{I}$ be the set of all ISets as defined in Def. 1. At any given time, only one ISet from each $\mathcal{I}^{O}$ can be activated.

2) No Coordination $(\mathrm{NC})$ : ON-OFF coordination involves a large set of independent sets (whose cardinality grows exponentially with the number of AIs). Such complexity might not always be desirable. In another extreme, we could employ no coordination at all. Under no coordination (NC), all transmit AIs in the network would stay scheduled all the time, as long as it is possible to do so. The only exception would be the case when a backhaul link and an access link in $\mathrm{RN} j$ are both operating on the same set of channels. In such a case, tAI of RN $j$ has to be turned-off when the backhaul link to $j$ is active. Such restrictions do not appear when $m \in T_{j}$ and $n \in R_{j}$ are allocated orthogonal set of channels. By restricting the set of ISets to a subset of $\mathcal{I}^{O}$ that satisfies this condition, we can define the set of ISets $\mathcal{I}^{N C}$ for NC.

\section{$B$. Flow routing under multi-association}

User association determines whether user $i$ is associated to BS $j$ or not. Conventionally, a user associates to only one BS (i.e., it is a single-association). However, in our model we allow a UE to associate to more than one BS (i.e., we allow multi-association). Multi-association allows us to obtain a tractable model. It has been shown in [9], [7] that it is possible to obtain a single-association whose performance is comparable to the upper-bound corresponding to multi-association. A user association structure in our twohop network is in fact embedded into a more general aspect, namely, the flow routing. Let $x_{l}^{f}$ represent the amount of flow $f$ routed through logical link $l$. Obtaining an optimal flow routing $\left\{x_{l}^{f}\right\}$ would automatically yield the optimal multiassociation.

\section{Problem Formulation}

Our aim is to obtain proportional fair throughput allocations $\left\{\lambda_{f}\right\}_{f \in \mathcal{F}}$ under optimal scheduling/transmission-coordination and flow-routing/user-association. Given a set of nodes $\mathcal{N}$, a set of flows $\mathcal{F}$, a set of bands $\mathcal{B}$, the associated channels 
and the rate functions, channel gain $G_{m, n}$ between any two AIs, a set of tAIs $T=\left\{T_{n}\right\}_{n \in \mathcal{N}}$ and rAIs $R=\left\{R_{n}\right\}_{n \in \mathcal{N}}$, their directivity properties $\left\{D_{m}(\theta)\right\}_{m \in T \cup R}$, the adjacency indicators $A[m, n]$, the channel allocations $C(\tilde{l})$ for all physical links, the power allocation $P(\tilde{l})$ for all physical links, the set of ISets $\mathcal{I}$ can be constructed a priori. Our problem of proportional fair throughput allocation under a joint optimal scheduling/coordination and flow-routing/user-association, can then be stated as follows.

$$
\begin{aligned}
& {[\mathbf{P}] \max \sum_{f \in \mathcal{F}} \log \left(\lambda_{f}\right)} \\
& \sum_{m \in T_{n}}\left(\sum_{l \in \mathcal{L}: o(l) \in m} x_{l}^{f}\right)-\sum_{m \in R_{n}}\left(\sum_{l \in \mathcal{L}: d(l) \in m} x_{l}^{f}\right) \\
& =\lambda_{f} \mathbf{1}_{\left\{n=f_{s}\right\}}-\lambda_{f} \mathbf{1}_{\left\{n=f_{d}\right\}}, \forall n \in \mathcal{N}, \forall f \in \mathcal{F} \\
& \sum_{f \in \mathcal{F}} x_{l}^{f} \leq|C(\tilde{l})| \sum_{s \in \mathcal{I}: l \in s} \alpha_{s} R(l), \quad \forall l \in \mathcal{L} \\
& \sum_{s \in \mathcal{I}} \alpha_{s} \leq 1 \\
& \alpha_{s} \geq 0, x_{l}^{f} \geq 0, \lambda_{f} \geq 0, \quad \forall s \in \mathcal{I}, \forall f \in \mathcal{F}, \forall l \in \mathcal{L}
\end{aligned}
$$

(6) is the flow-conservation constraint. (7) is the capacity constraint that limits the total amount of flow in a link $l$. (8) is the scheduling constraint. Maximizing the objective $\sum_{f \in \mathcal{F}} \log \left(\lambda_{f}\right)$ is known to yield the proportional fair throughput allocation [12]. A PF throughput allocation is known to maximize the geometric mean (GM) throughput $\left(\prod_{f \in \mathcal{F}} \lambda_{f}\right)^{1 /|\mathcal{F}|}$ and hence we will use GM throughput as the performance metric.

\section{SCENARIOS}

We assume that Band 1 is an LTE band with $M_{T}$ LTE OFDM channels available for the entire multi-cell system. Further, we assume that all user links operate only in this band and thus a UE is equipped with an LTE AI, exclusively used as an rAI. Let the rAI for UE $i$ be simply referred to as $i$ for all $i \in \mathcal{U}$. The set of LTE channels available for user links are allocated to different macro-cells by employing a reuse factor of 3 .

We consider three scenarios. Scenario 1 corresponds to wired backhauling. The other two scenarios correspond to wireless backhauling. In the first of the two wireless backhauling scenarios (Scenario 2), the backhauling is done on the same band as the user links (i.e., $S=1$ ) while in the second (Scenario 3), backhaul links use a dedicated band. Scenario 3 is similar in many ways to Scenario 1 . Scenario 2 on the other hand involves a number of different configurations. Thus, we first discuss Scenarios 1 and 3.

\section{A. Scenario 1: Wired Scenario}

This scenario consists of one LTE band (i.e., $S=1$ ), and one omni directional LTE AI at the MBS as well as at each $\mathrm{SC}$, used for the direct and the access links, respectively. In addition, there are $X$ wired backhaul links, each with a capacity of $C$. Since all $M_{T}$ LTE channels are available for user links, a given macro-cell gets a pool of LTE channels
$\mathcal{M}^{(1)}$ simply written as $\mathcal{M}$ with $|\mathcal{M}|=\frac{M_{T}}{3}=M$. We have already presented models for this scenario in [9] for two channel allocation schemes, namely, co-channel deployment (CCD) and orthogonal deployment (OD). In OD, the direct and the access links operate on orthogonal channels (respectively, $M-K$ and $K$ channels) whereas in CCD both the direct and the access links are allocated the entire set of $M$ LTE channels. Thus, in CCD, the direct and the access links interfere with each other. We had shown in [9] that in the absence of ONOFF coordination, OD outperforms CCD, provided that the channel split parameter $K$ is selected optimally. Note that with sufficiently large $C$, the wired scenario represents an upper bound on performance for our HetNet. In this study, we will use the performance of the wired scenario with optimal OD without coordination (OD-NC) as a benchmark.

\section{B. Scenario 3: Dedicated-band relay scenario}

In this scenario, in addition to the LTE band (Band 1) for the user links, a separate mmWave band (Band 2) is available exclusively for the backhaul links (i.e., $S=2$ ). We assume that the mmWave band has a bandwidth of $F \mathrm{MHz}$. In order to exploit this new band, the MBS needs to have at least one additional $\mathrm{AI}$ in the mmWave band and each $\mathrm{RN}$ needs to have one additional AI for receiving on the mmWave band. We consider two configurations for this scenario:

1) mmWave-TDM: MBS has one omnidirectional mmWave air-interface for transmitting to all backhaul links. Thus, the backhaul links operate in a time-shared fashion. We assume that all backhaul links in a given macro-cell operate with a reuse factor of 3 (to manage the interference), and thus get a mmWave bandwidth of $B=\frac{F}{3}$.

2) mmWave-SIMUL: MBS has one directional mmWave airinterface for each backhaul link. Thus, the backhaul links operate as narrow-beam simultaneous links, all operating on the mmWave band (Band 2). In this case, we assume that the mmWave links do not interfere with each other, since the beams are very narrow. Hence, we can exploit full reuse, i.e., a backhaul link operates in entire mmWave band, i.e., $B=F$.

The mmWave band is assumed to comprise of one wideband channel of bandwidth $B$ and a logarithmic rate function, $f^{(2)}(\gamma)=B \log (1+\gamma)$. This scenario is very similar to Scenario 1 in the sense that the backhaul links do not steal channel resources from the user links. Thus, both channel allocations, OD and CCD, as defined before are relevant. Similar to the wired scenario, we consider OD only. However, unlike the wired scenario, the available transmit power budget at the MBS has to be divided between the direct links and the backhaul links. Let $P_{B}$ be the power allocated to each mmWave backhaul link, then the power allocated to direct links will be $P_{M}-P_{B}$ for mmWave-TDM and $P_{M}-X P_{B}$ for mmWave-SIMUL. In other words, the values of $P_{B}$ and $K$ completely characterize the channel allocation and power allocation, which can be used to obtain the best GM throughput $\Lambda^{*}\left(P_{B}, K\right)$. The best performance can then be obtained by fine tuning the power and channel allocations:

$$
\max _{P_{B} \in P_{\Delta}, K \in\{1,2, \cdots, M\}} \Lambda^{*}\left(P_{B}, K\right)
$$

\section{Scenario 2: User-band relay scenario}

In this scenario, $S=1$ and hence the backhaul links have to operate on the same LTE band as user links. We assume 


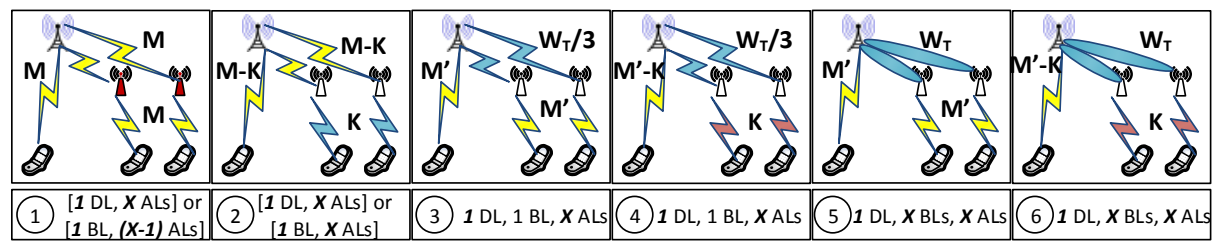

Fig. 2: Configurations of Scenario 2 (DL: Direct Link, AL: Access Link, BL: Backhaul Link)

TABLE I: Model parameters for Scenario 2 configurations

\begin{tabular}{|c|c|c|c|c|c|}
\hline Number of tAIs & Config. & Channels $C(\tilde{l})$ & Channel allocation constraints & $P(\tilde{l})^{\dagger}$ & $V_{i}$ \\
\hline \multirow[t]{4}{*}{1 AI: } & 1 & $\begin{array}{l}=\mathcal{M} \\
\forall \tilde{l} \in \mathbf{L}_{D} \cup \mathbf{L}_{A} \cup \mathbf{L}_{B}\end{array}$ & $|\mathcal{M}|=\frac{M_{T}}{3}=M$ & $\begin{array}{l}=P_{M} \\
\forall \tilde{l} \in \mathbf{L}_{D} \cup \mathbf{L}_{B}\end{array}$ & (Given) \\
\hline & 2 & $\begin{array}{l}=\mathcal{M}_{1}, \forall \tilde{l} \in \mathbf{L}_{D} \cup \mathbf{L}_{B} \\
=\mathcal{M}_{2}, \forall \tilde{l} \in \mathbf{L}_{A}\end{array}$ & $\begin{array}{c}\frac{M_{T}}{3}=M ;\left|\mathcal{M}_{2}\right|=K \\
\left|\mathcal{M}_{1}\right|=M-K ; \mathcal{M}_{1} \cap \mathcal{M}_{2}=\emptyset\end{array}$ & $\begin{array}{l}=P_{M} \\
\forall \tilde{l} \in \mathbf{L}_{D} \cup \mathbf{L}_{B}\end{array}$ & $(K \in[1, M])$ \\
\hline & 3 & $\begin{array}{l}=\mathcal{M}_{1}, \forall l \in \mathbf{L}_{D} \cup \mathbf{L}_{A} \\
=\mathcal{M}_{2}, \forall \tilde{l} \in \mathbf{L}_{B}\end{array}$ & $\begin{array}{c}\mathcal{M}_{1} \cap \mathcal{M}_{2}=\emptyset \\
\left|\mathcal{M}_{2}\right|=\frac{W_{T}}{3} ;\left|\mathcal{M}_{1}\right|=\frac{M_{T}-W_{T}}{3}=M^{\prime}\end{array}$ & $\begin{array}{l}=P_{M}-P_{B}, \forall l \in \mathbf{L}_{D} \\
=P_{B}, \forall \tilde{l} \in \mathbf{L}_{B}\end{array}$ & $\begin{array}{c}\left(W_{T} \in\left[1, M_{T}\right]\right. \\
\left.\quad P_{B} \in P_{\Delta}\right) \\
\end{array}$ \\
\hline & 4 & $\begin{array}{l}=\mathcal{M}_{1}, \forall \tilde{l} \in \mathbf{L}_{D} \\
=\mathcal{M}_{2}, \forall \tilde{l} \in \mathbf{L}_{B} \\
=\mathcal{M}_{3}, \forall \tilde{l} \in \mathbf{L}_{A}\end{array}$ & $\begin{array}{c}\mathcal{M}_{j} \cap \mathcal{M}_{j^{\prime}}=\emptyset, \forall j, j^{\prime} \in\{1,2,3\}, j \neq j^{\prime} \\
\left|\mathcal{M}_{2}\right|=\frac{W_{T}}{3} ; M^{\prime}=\frac{M_{T}-W_{T}}{{ }^{3}} \\
\left|\mathcal{M}_{3}\right|=K ;\left|\mathcal{M}_{1}\right|=M^{\prime}-K\end{array}$ & $\begin{array}{l}=P_{M}-P_{B}, \forall \tilde{l} \in \mathbf{L}_{D} \\
=P_{B}, \forall \tilde{l} \in \mathbf{L}_{B}\end{array}$ & $\begin{array}{c}\left(W_{T} \in\left[1, M_{T}\right]\right. \\
P_{B} \in P_{\Delta} \\
\left.K \in\left[1, M^{\prime}\right]\right)\end{array}$ \\
\hline \multirow[t]{2}{*}{$X+1$ AIs: } & 5 & $\begin{array}{l}=\mathcal{M}_{1}, \forall \tilde{l} \in \mathbf{L}_{D} \cup \mathbf{L}_{B} \\
=\mathcal{M}_{2}, \forall \tilde{l} \in \mathbf{L}_{A}\end{array}$ & $\begin{array}{c}\mathcal{M}_{1} \cap \mathcal{M}_{2}=\emptyset \\
\left|\mathcal{M}_{2}\right|=W_{T} ;\left|\mathcal{M}_{1}\right|=\frac{M_{T}-W_{T}}{3}=M^{\prime}\end{array}$ & $\begin{array}{l}=P_{M}-X P_{B}, \forall \tilde{l} \in \mathbf{L}_{D} \\
=P_{B}, \forall \tilde{l} \in \mathbf{L}_{B}\end{array}$ & $\begin{array}{l}\left(W_{T} \in\left[1, M_{T}\right]\right. \\
\left.\quad P_{B} \in P_{\Delta}\right)\end{array}$ \\
\hline & 6 & $\begin{array}{l}=\mathcal{M}_{1}, \forall \tilde{l} \in \mathbf{L}_{D} \\
=\mathcal{M}_{2}, \forall \tilde{l} \in \mathbf{L}_{B} \\
=\mathcal{M}_{3}, \forall \tilde{l} \in \mathbf{L}_{A}\end{array}$ & $\begin{array}{c}\mathcal{M}_{j} \cap \mathcal{M}_{j^{\prime}}=\emptyset, \forall j, j^{\prime} \in\{1,2,3\}, j \neq j^{\prime} \\
\left|\mathcal{M}_{2}\right|=W_{T} ; M^{\prime}=\frac{M_{T}-W_{T}}{3} \\
\left|\mathcal{M}_{3}\right|=K ;\left|\mathcal{M}_{1}\right|=M^{\prime}-K\end{array}$ & $\begin{array}{l}=P_{M}-X P_{B}, \forall \tilde{l} \in \mathbf{L}_{D} \\
=P_{B}, \forall \tilde{l} \in \mathbf{L}_{B}\end{array}$ & $\begin{array}{c}\left(W_{T} \in\left[1, M_{T}\right]\right. \\
P_{B} \in P_{\Delta} \\
\left.K \in\left[1, M^{\prime}\right]\right) \\
\end{array}$ \\
\hline
\end{tabular}

that an SC has one omni AI that it uses for both, transmitting on an access link as well as receiving on the backhaul link. Note that this means an SC cannot simultaneously transmit and receive in the same set of channels (even though it can do so over orthogonal set of channels). If an SC had two AIs, such limitation could be avoided. However, in the absence of a mechanism to separate the interference between a tAI and an rAI of the same node (e.g., interference cancellation, spatial separation), the additional AI would not be beneficial.

We consider six configurations for this scenario which differ in terms of the number of AIs at the MBS and the way the LTE channels are allocated to the direct, access, and the backhaul links. In other words, each configuration is characterized by a given number of AIs at the MBS and the channel allocation scheme. The configurations are depicted in Fig. 2. Even though our selection of configurations is not exhaustive, we believe that we have included the most natural ones. Next, we discuss the implications of having a number of AIs at the MBS as well as the choice that we make in terms of channel allocation. 1) Number of AIs: In terms of the number of AIs at the MBS, we consider two possibilities: $1 \mathrm{AI}$ and $X+1$ AIs (recall that $\mathrm{X}$ is the number of SCs). We could also consider the case with 2 omni AIs, one for the direct links and the other for the backhaul links. However, having a simultaneous direct and backhaul link on the same set of channels would mean a lot of mutual interference due to the omni directional nature of both AIs.

- 1 AI: The MBS has only one omni AI. This AI is used for both the direct and the backhaul links. This means that on a given channel, only one link can be activated at a time. Configurations 1 to 4 in Fig. 2 are such configurations. Let $o_{0}$ be the omni AI of the MBS, and let $T_{j}$ and $R_{j}$ respectively be the tAI and rAI in $\mathrm{RN} j$, then the set of direct links is given as $\mathbf{L}_{D}=\left\{\left(o_{0}, i\right): i \in \mathcal{U}\right\}$, the set of access links is given as
$\mathbf{L}_{A}=\left\{\left(T_{j}, i\right): j \in \mathcal{P}, i \in \mathcal{U}\right\}$, and the set of backhaul links is given as $\mathbf{L}_{B}=\left\{\left(o_{0}, R_{j}\right): j \in \mathcal{P}\right\}$.

- $X+1$ AIs: The MBS has one omni AI called $o_{0}$ for the direct links, and one directional AI $D_{0 j}$ for each backhaul link. This means that on a given channel, up to $X+1$ links can be activated simultaneously. Configurations 5 and 6 in Fig. 2 are such configurations. The set of direct, access, and backhaul links are then given as $\mathbf{L}_{D}=\left\{\left(o_{0}, i\right): i \in \mathcal{U}\right\}$, $\mathbf{L}_{B}=\left\{\left(D_{o j}, R_{j}\right): j \in \mathcal{P}\right\}$, and $\mathbf{L}_{A}=\left\{\left(T_{j}, i\right): j \in \mathcal{P}, i \in\right.$ $\mathcal{U}\}$ respectively.

2) Channel and power allocation:

In addition to a given number of AIs at the MBS, each configuration in Fig. 2 has a specific channel allocation, which is illustrated in Fig. 2 and specified in details in Table I. For Configuration 1, all (direct, access and backhaul) links are allocated all the available channels $(\mathcal{M})$. For Configuration 2 , on the other hand, the direct and the backhaul links are allocated the same set of channels whereas the access links are allocated the remaining channels. Table I also shows the power allocated to each link.

Each channel allocation choice has its own impact:

Is a direct link orthogonal to an access link? If no, access links will receive large interference from the direct links and thus some transmission coordination (i.e., ON OFF TC) might be required. Configurations 1,3 and 5 are such configurations where direct and access links interfere and thus we study both the NC and ON-OFF TC. For Configurations 2, 4 and 6, however, we only consider the case of no coordination.

Is a backhaul link orthogonal to a direct link? If yes, a backhaul link can operate in parallel to a direct link (Configurations 3 to 6). In that case, the MBS can simultaneously have one direct link and either one backhaul link (for configurations with $1 \mathrm{AI}$ at MBS) or $X$ backhaul links (for configurations with $X+1$ AIs at MBS). In this case, power allocation for the 
backhaul links is crucial. Let $P_{B}$ be the power allocated to a backhaul link. Then, the power allocated to each direct link is $P_{M}-P_{B}$ for Configurations 3 and 4 and it is $P_{M}-X P_{B}$ for Configurations 5 and 6.

Is a backhaul link orthogonal to an access link? If no, an RN cannot transmit while it is receiving on the backhaul link (e.g., Configuration 1). Configuration 1 is an example of the in-band RN deployment specified in LTE-A [2].

Note that, for each configuration, given the channel and power allocation per physical link, our optimization model $[\mathbf{P}]$ can be used to obtain the optimal geometric mean throughput. Determining a channel allocation and power allocation per physical link requires a number of parameters to be fixed. For configuration $i$, let $V_{i}$ be the set of these parameters (as shown in Table I). For example, for Configuration 1, there are no such parameters (in the sense that no channel/power allocation parameter has to be chosen). For Configuration 6 on the other hand, there are three parameters (namely, the number of channels allocated to backhaul links $\left(W_{T}\right)$, the channelsplit parameter between direct and the access links $(K)$, and the power allocated to the backhaul links $\left.P_{B}\right)$. Let $\Lambda_{i}^{*}\left(V_{i}\right)$ be the optimal GM throughput obtained for a given choice of channel and power allocation $V_{i}$, then the best performance for configuration $i$ is obtained by fine-tuning these parameters: $\Lambda_{i}^{*}=\max _{V_{i}} \Lambda_{i}^{*}\left(V_{i}\right)$.

\section{Numerical Results}

We take an ISD of $1732 \mathrm{~m}$., which corresponds to a rural settings. The central macro-cell in Fig. 1 forms the HetNet system with its centrally placed MBS and $X=4 \mathrm{SCs}$ at a radius of $d=400 \mathrm{~m}$., symmetrically. $N=50$ users are uniformly distributed in the central cell. A given realization $i$ of user positions (and the corresponding channel-gains across all communication links) is taken as a static snap-shot of the system. We study 100 such realizations, with a condition that each of them is connected even when the macro cell does not have any SCs. The physical layer parameters for LTE (shown in Table III) correspond to the parameters recommended on the 3GPP evaluation recommendations [2]. The LTE path-loss models for MBS and small cells are used along with a lognormal shadowing of $8 \mathrm{~dB}$ standard deviation, for generating channel-gains $G_{j i}$ for the direct and the access links.

We assume that the relays are outdoor and thus there is no penetration loss (pen. loss) for backhaul links. Also, we assume that there exists a line-of-sight (LOS) between the MBS and an RN of the same cell and thus we take LOS pathloss model between the serving MBS and its RNs. We use non-LOS (NLOS) path-loss model to compute channel gains between an MBS and an RN that are located in different cells (i.e., for calculating interference). Directional backhaul links have an additional directional gain of $20 \mathrm{~dB}$ and we assume that the directional links do not interfere with each other.

Also, while calculating inter-cell interference, due to the small transmit power of SCs and a much faster power attenuation with distance, we ignore the interference from SCs in nearby macrocells. We however account for the interference from all surrounding MBSs.

We use an MCS with 15 rates for the user links [9]. For the LTE relay backhaul links (Scenario 2), we have two extra
TABLE III: Physical layer parameters

\begin{tabular}{|c|c||c|c|}
\hline UE Noise Power & $-174 \mathrm{dBm} / \mathrm{Hz}$ & $P_{M}$ & $43 \mathrm{dBm}$ \\
\hline$P_{S}$ & $30 \mathrm{dBm}$ & Channel BW & $180 \mathrm{KHz}$ \\
\hline UE Noise-figure & $9 \mathrm{~dB}$ & RN Noise-figure & $5 \mathrm{~dB}$ \\
\hline UE Pen. Loss & $20 \mathrm{~dB}$ & & \\
\hline MBS Ant. Gain & $15 \mathrm{dBi}$ & SC Ant. Gain & $5 \mathrm{dBi}$ \\
\hline Directional Gain & $20 \mathrm{dBi}$ & $T_{\text {subframe }}$ & $1 \mathrm{~ms}$ \\
\hline$n_{s c}$ & 12 & $n_{t s}$ & 14 \\
\hline MBS-UE Path-loss & $128.1+37.6 \log _{10}(d / 1000), d \geq 35 m$ \\
\hline SC-UE Path-loss & $140.7+36.7 \log _{10}(d / 1000), d \geq 10 m$ \\
\hline MBS-SC Path-loss & LOS: $103.4+24.2 \log _{10}(d / 1000)$ \\
\hline NLOS: $131.1+42.8 \log _{10}(d / 1000)$ \\
\hline mmWave: & $25 \mathrm{dBi}$ & Rcv. Gain & $12 \mathrm{dBi}$ \\
\hline Tr. Gain & $3 \mathrm{~dB}$ & Noise-figure & $7 \mathrm{~dB}$ \\
\hline Impl. Loss & \multicolumn{3}{|c|}{$157.4+32 \log _{10}(d / 1000)$} \\
\hline Path-loss & $P_{\Delta}=\{-10,-5,0,5,10,15,20,25,30\} \mathrm{dBm}$ \\
\hline
\end{tabular}

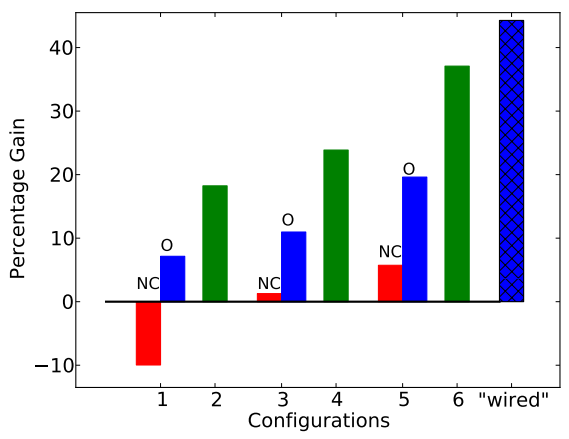

Fig. 3: Scenario 2: Different configurations (NC means no coordination, O means ON-OFF coordination)

modulation schemes (corresponding to 256QAM with a rate of $1 / 2$ and 2/2) (see Table II). The efficiency $\eta$ is related to rate $R$ as $R=\eta \frac{n_{s c} n_{t s}}{T_{s y m b o l}}$ where $n_{s c}$ is the number of sub-carriers per OFDM symbol, $n_{t s}$ is the number of time-slots in one OFDM subframe, and $T_{\text {symbol }}$ is the duration of one OFDM subframe.

The mmWave parameters are taken from [6] and are shown in Table III. The path-loss model taken is considered to be a realistic model for links at $28 \mathrm{GHz}$. As mentioned already, a logarithmic rate function is assumed for the mmWave links.

For a given backhauling scenario and a specific configuration, we use our optimization framework to obtain the allocated throughputs for each realization $i$ and obtain the best GM throughput by fine-tuning the channel and power allocations as explained before. Also, we take the scenario of MBS-only deployment as the base scenario and benchmark the different scenarios and their configurations in terms of the gain in performance w.r.t. that MBS-only deployment.

\section{A. Scenario 2: User-band relay scenario}

Fig. 3 shows the percentage gain in GM throughput (with respect to the MBS-only case) for each of the six configurations of the user-band relay scenario as well as the wired scenario. The results show that Configurations 1, 3 and 5 (all corresponding to configurations where the access links get interference from the direct links) in the absence of interference coordination (NC) do not yield meaningful throughput gains. In fact, with Configuration 1 , there is a negative gain in the performance w.r.t. the MBS-only case. This means the spatial reuse gain and SINR improvement brought to some poor users 
TABLE II: Available rates and the corresponding SNR thresholds

\begin{tabular}{|c|c|c|c|c|c|c|c|c|c|c|c|c|c|c|c|c|c|}
\hline Threshold SNR (dB) & -6.5 & -4 & -2.6 & -1 & 1 & 3 & 6.6 & 10 & 11.4 & 11.8 & 13 & 13.8 & 15.6 & 16.8 & 17.6 & 21.04 & 24.07 \\
\hline Efficiency (bits/symbol) $(\eta)$ & 0.15 & 0.23 & 0.38 & 0.60 & 0.88 & 1.18 & 1.48 & 1.91 & 2.41 & 2.73 & 3.32 & 3.9 & 4.52 & 5.12 & 5.55 & 7.0 & 8.0 \\
\hline
\end{tabular}

does not off-set the loss in performance due to an overall increased interference. Even for the configuration with $X+1$ AIs (Configuration 5), a very small gain in performance is observed. These configurations (1, 3 and 5), however do much better in the presence of ON-OFF coordination. The results are not surprising since ON-OFF coordination is a means to combat the interference to an access link from a direct link.

The figure also shows performance results for Configurations 2, 4 and 6 without coordination. These configurations do not require the transmission coordination for protecting access links from the MBS interference. The performance of these configurations show that the number of AIs and the channel allocation scheme play a very important role in the performance of an RN deployment. With $X+1$ AIs and an appropriate channel allocation (Configuration 6), we obtain performance not very far from the upper bound $(38 \%$ for Configuration 6 , and $44 \%$ for the upper bound).

\section{B. Scenario 3: mmWave backhaul}

In Fig. 4a, we plot the GM Throughput performance of two configurations of mmWave backhauling (mmWave-SIMUL and $\mathbf{m m W a v e - T D M}$ ), as a function of the available mmWave bandwidth $F$, assuming that the best power allocation $P_{B}$ in $P_{\Delta}$ is chosen. We also show the upper-bound which corresponds to the wired scenario with infinite backhaul capacities. As we can see, a bandwidth of about $2.5 \mathrm{MHz}$ can yield a performance within $98 \%$ of the upper-bound. This is a very small bandwidth in a typical mmWave spectrum. This shows that a small fraction of available mmWave bandwidth can be sufficient to satisfy the load on backhaul links.

In Fig. 4b, we plot the GM throughput versus the backhaul link capacity for the wired scenario, and for the two values of bandwidth of mmWave-SIMUL. For the mmWave scenario, the backhaul capacity is determined by the power $P_{B}$ we allocate to the backhaul links. For the wired deployment, it is obvious that the performance improves with an increase in backhaul capacity before it saturates. For the mmWave deployment, however, the results show that it is important to make sure that the relay backhaul power $P_{B}$ is carefully chosen, or otherwise the performance can degrade significantly.

\section{CONCLUSION}

We formulated an optimization framework to model and evaluate different configurations of relay node deployment in a HetNet consisting of multiple bands and air interfaces per node. Our results show that some configurations of user-band relay scenario can yield negative or negligible performance gains where as some others can yield very good performances. Also, our results show that for the dedicated-band relay scenario, a small mmWave bandwidth is sufficient to satisfy the load on a typical small-cell backhaul, provided that available parameters are chosen carefully. Thus, it is quasi-equivalent to the wired scenario.

\section{REFERENCES}

[1] A. Damnjanovic et al., "A survey on 3GPP heterogeneous networks," IEEE Wireless Commun. Mag., vol. 18, no. 3, pp. 10 -21, June 2011.

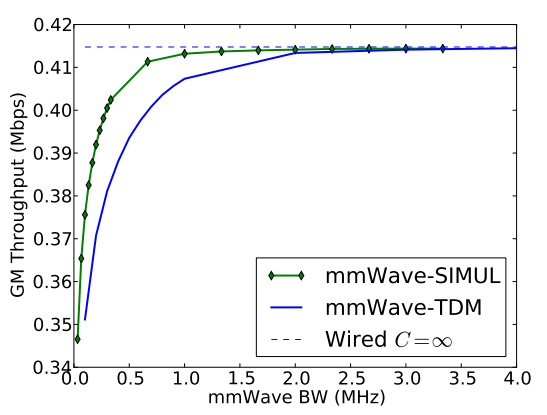

(a) GM Throughput vs. $F$

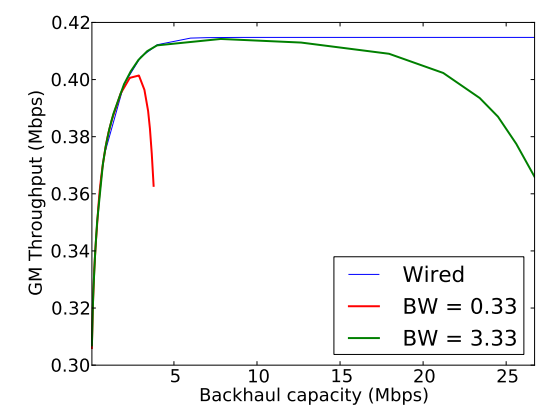

(b) GM Throughput vs. backhaul link capacities

Fig. 4: Scenario 3 (mmWave) along with Scenario 1 (Wired)

[2] 3GPP, "TR 36.814 v9.0.0: Further advancements for E-UTRA physical layer aspects (release 9)," March 2010.

[3] D. Bultmann, T. Andre, and R. Schoenen, "Analysis of 3GPP LTEAdvanced cell spectral efficiency," in Personal Indoor and Mobile Radio Communications (PIMRC), 2010 IEEE 21st International Symposium on, 2010, pp. 1876-1881.

[4] J. Gora and S. Redana, "In-band and out-band relaying configurations for dual-carrier LTE-Advanced system," in Personal Indoor and Mobile Radio Communications (PIMRC), 2011 IEEE 22nd International Symposium on, 2011, pp. 1820-1824.

[5] T. M. de Moraes, M. D. Nisar, A. A. Gonzalez, and E. Seidel, "Resource allocation in relay enhanced LTE-Advanced networks," EURASIP Journal on Wireless Communications and Networking, vol. 2012, no. 1, pp. $1-12,2012$.

[6] S. Rajagopal, S. Abu-Surra, Z. Pi, and F. Khan, "Antenna array design for multi-gbps mmwave mobile broadband communication," in Global Telecommunications Conference (GLOBECOM), 2011 IEEE, pp. 1-6.

[7] D. Fooladivanda and C. Rosenberg, "Joint resource allocation and user association for heterogeneous wireless cellular networks," IEEE Trans. Wireless Commun., vol. 12, no. 1, pp. 248-257, 2013.

[8] R. Madan, J. Borran, A. Sampath, N. Bhushan, A. Khandekar, and T. Ji, "Cell association and interference coordination in heterogeneous LTE-A cellular networks," IEEE J. Sel. Areas Commun., vol. 28, no. 9, pp. 1479 -1489 , december 2010.

[9] J. Ghimire and C. Rosenberg, "Resource allocation, transmission coordination and user association in heterogeneous networks: A flow-based unified approach," IEEE Trans. Wireless Commun., vol. 12, no. 3, pp. 1340-1351, 2013.

[10] — "On the need for coordination among base stations in a heterogeneous network," in Modeling and Optimization in Mobile, Ad Hoc and Wireless Networks (WiOpt), 2012 10th International Symposium on. IEEE, 2012, pp. 254-261.

[11] R. Sappidi, S. Mosharrafdehkordi, C. Rosenberg, and P. Mitran, "Planning for small cells in a cellular network: why it is worth it," in IEEE Wireless Commun. and Networking Conference (WCNC), April 2014.

[12] F. Kelly, "Charging and rate control for elastic traffic," European transactions on Telecommunications, vol. 8, no. 1, pp. 33-37, 1997. 